Trauma Berufskrankh 2005 · 7[Suppl 1]:S58-S64 DOI 10.1007/s10039-004-0918-1

Online publiziert: 12 . August 2004

(c) Springer-Verlag 2004

M. E. Wenzl ${ }^{1} \cdot$ S. Fuchs ${ }^{2} \cdot$ D. Wolter ${ }^{2} \cdot$ C. Jürgens ${ }^{2}$

${ }^{1}$ Klinik für Unfallchirurgie, Universitätsklinikum Schleswig-Holstein, Campus Lübeck

${ }^{2}$ Klinik für Unfall- und Wiederherstellungschirurgie, BG-Unfallkrankenhaus, Hamburg

\title{
Distale Tibiafrakturen
}

\section{Erfahrungen mit einem winkelstabilen Plattensystem}

tiven randomisierten Studie, in der sie bei 50 Patienten einen transtendinösen mit einem paratendinösen Zugang verglichen, dass $70 \%$ der Patienten auch nach der Marknagelentfernung noch Beschwerden hatten, die in 50\% der Fälle zu relevanten Beeinträchtigungen führten. Dieses Problem, das meist fernab der ursprünglichen Verletzungsregion liegt, tritt nach Plattenosteosynthesen nicht auf.

Die Plattenosteosynthese wird in letzter Zeit aufgrund der minimalinvasiven Implantationstechniken wieder vermehrt auch an der Tibia eingesetzt $[6,8]$.

Über die Anwendung winkelstabiler interner Plattenfixateure in Verbindung mit minimalinvasiven Implantationstechniken wurden bisher nur erste klinische Erfahrungen publiziert $[5,11]$. Im Folgenden wird über die Ergebnisse mit einem winkelstabilen Plattensystem (TiFix, Fa. Litos Hamburg) in der Therapie von Frakturen und Pseudarthrosen der distalen Tibia berichtet.

\section{Material und Methode}

Bei dem verwendeten TiFix-System handelt es sich um einen multidirektional winkelstabilen Plattenfixateur interne aus

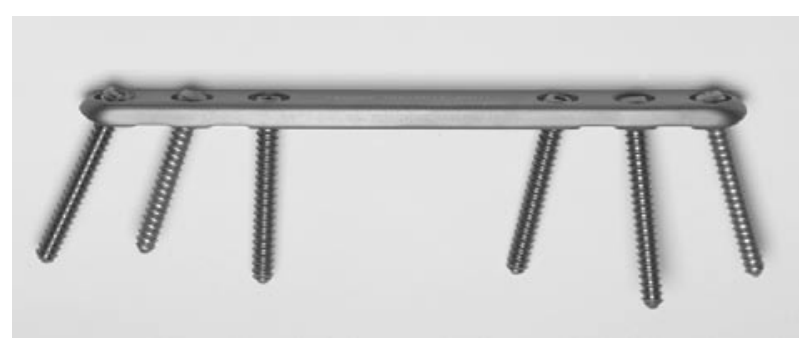

Abb. $1<$ Multidirektional winkelstabiler Titanfixateur interne für den Unterschenkel (TiFix)
Reintitan. Die winkelstabilen Schrauben können bis zu Winkeln von $40^{\circ}$ zur Plattenquer- und -längsachse frei platziert werden [25] (• Abb. 1).

Von September 1997-Dezember 2000 wurden 41 Frakturen und 38 Pseudarthrosen der Tibia bei 76 Patienten mit diesem System stabilisiert, wobei das distale Tibiadrittel mit mehr als 50\% der Implantationen die Hauptlokalisation darstellte (• Tabelle 1).

Bei mehr als der Hälfte der Patienten lagen offene Frakturen vor. Bei 30\% mussten Begleitverletzungen therapiert werden. Bei den 25 distalen Frakturen fanden sich in 5 Fällen Frakturausläufer bis in das obere Sprunggelenk.

Für die operative Versorgung aller Tibiafrakturen mit dem TiFix-System wurde folgendes Konzept entwickelt und konsequent befolgt:

- Geschlossene Frakturen ohne wesentlichen Weichteilschaden wurden primär stabilisiert. Die Reposition erfolgte geschlossen (ggf. wurde temporär intraoperativ ein Fixateur externe angelegt). Der TiFix wurde minimalinvasiv in der Untertunnelungstechnik über 2 kleine Hautinzisionen eingeschoben und an der medialen Tibiafacette nach entsprechender Anpassung der Plattenkontur mit 6 winkelstabilen Schrauben fixiert.

- Offene und geschlossene Frakturen mit drittgradigen Weichteilschäden wurden primär im Fixateur externe stabilisiert. Falls sich noch eine korrekturbedürftige Fehlstellung zeigte, wurde mit dem Hexapodensystem [21] eine suk- 
zessive Fehlstellungskorrektur durchgeführt. Nach Abheilung oder plastischer Deckung der Hautweichteilschäden wurden - bei reizlosen Eintrittsstellen der Fixateurpins - in einer Sitzung der TiFix in minimalinvasiver Technik bei noch liegendem externem Fixateur implantiert und dieser anschließend entfernt. Lag der Fixateur externe über mehr als 3 Wochen ein oder waren die Pineintrittsstellen entzündet, wurde ein fixateurfreies Intervall bis zum Abklingen der Entzündungszeichen zwischengeschaltet (Minimum 9 Tage).

Bei der folgenden klinischen Auswertung werden nur noch die distalen Tibiafrakturen und -pseudarthrosen berücksichtigt.

\section{Distale Tibiafrakturen und -pseudarthrosen}

Hier waren Primärimplantationen nur bei $40 \%$ der Patienten möglich. In 6 von 15 Fällen erschien ein direkter Umstieg vom Fixateur externe auf einen TiFix zu riskant (- Tabelle 2).

In der Gruppe der Pseudarthrosen waren in $75 \%$ der Fälle offene Frakturen vorausgegangen, woraus sich die hohe Anzahl von primären Fixateur-externe-Behandlungen erklärt.

In 50\% der Fälle war es zur distalen Tibiafraktur im Rahmen von Mehrfachverletzungen bzw. Polytraumen gekommen, initial lagen bei 20\% der Patienten Nervenschäden vor. Die Anzahl der Voroperationen war mit durchschnittlich 3,4 sehr hoch (- Tabelle 3).

Bis auf 3 von 20 Fällen war auch bei den Pseudarthrosen eine minimalinvasive Operationstechnik möglich. Grundsätzlich wurde autologe Spongiosa vom vorderen oder hinteren Beckenkamm transplantiert. Bei der regelhaft durchgeführten bakteriologischen Untersuchung des Pseudarthrosengewebes fand sich bei 3 von 19 untersuchten Präparaten ein positiver Keimbefund (15,8\%)!

In mehr als der Hälfte der Fälle wurden bei den distalen Tibiafrakturen und pseudarthrosen zur Infektprophylaxe Septopalketten an die Platten angelegt, die bis zur Materialentfernung verblieben.

Die Nachbehandlung erfolgte in allen Fällen frühfunktionell unter Teilbelastung

Tabelle 1

Patientendaten bei Tibiafrakturen

\begin{tabular}{|c|c|c|c|}
\hline & Proximal & Diaphyse & Distal \\
\hline Zeitraum der Versorgung & 9/99-12/00 & $10 / 98-12 / 00$ & $11 / 97-12 / 00$ \\
\hline Patientenzahl & $8\left(39,50^{7}\right)$ & $6\left(19,50^{7}\right)$ & $24\left(99,15 \sigma^{7}\right)$ \\
\hline Anzahl Frakturen & 8 & 8 & 25 \\
\hline \multicolumn{4}{|l|}{ Alter [Jahre] } \\
\hline - Durchschnitt & 39,3 & 29,13 & 44,1 \\
\hline -Spannweite & $17-62$ & $17-51$ & $19-70$ \\
\hline \multicolumn{4}{|l|}{ Klassifikation nach AO } \\
\hline •A1-3 & 4 & 4 & 19 \\
\hline •B1-3 & 0 & 2 & 0 \\
\hline$\cdot C 1-3$ & 4 & 2 & 6 \\
\hline Weichteilschaden geschlossen & 3 & 2 & 15 \\
\hline \multicolumn{4}{|l|}{ Weichteilschaden offen } \\
\hline - Grad 1 & 2 & 3 & 4 \\
\hline - Grad 2 & 1 & 2 & 5 \\
\hline - Grad 3 & 2 & 1 & 1 \\
\hline Primärer Gefäß-Nerven-Schaden & 2 & 3 & 1 \\
\hline \multicolumn{4}{|l|}{ Unfallart } \\
\hline - Monotrauma & 4 & 3 & 18 \\
\hline - Mehrfachverletzung & 1 & 2 & 1 \\
\hline - Polytrauma & 3 & 1 & 5 \\
\hline \multicolumn{4}{|l|}{ Unfallursache } \\
\hline -Verkehr & 5 & 5 & 5 \\
\hline - Arbeit & 2 & 0 & 8 \\
\hline - Sport & 0 & 0 & 1 \\
\hline • Stürze & 1 & 3 & 11 \\
\hline
\end{tabular}

der verletzten Extremität anfänglich mit 10 kg Körpergewicht, dann abhängig vom radiologischen Befund mit sukzessiver Belastungssteigerung.

\section{Ergebnisse}

Alle 44 Patienten konnten nach durchschnittlich 22,4 Monaten (9-48 Monaten) nachuntersucht werden (Nachuntersuchungsquote: $100 \%$ ).

\section{Ausheilungsergebnisse}

Sämtliche Frakturen und Pseudarthrosen waren zu diesem Zeitpunkt knöchern durchbaut. Bei einem Patienten mit einer Infektpseudarthrose musste nach der Primäroperation bei verzögertem Durchbau noch eine weitere autologe Spongiosaplastik durchgeführt werden. Zum Zeitpunkt der 2. Spongiosaplastik zeigte sich kein Infekt mehr.

Vollbelastung war bei den Frakturen nach durchschnittlich 15,2 Wochen (8-
26 Wochen) und bei den Pseudarthrosen nach durchschnittlich 17,1 Wochen (852 Wochen) erreicht.

Die Beweglichkeit im oberen Sprunggelenk betrug nach Frakturstabilisierung durchschnittlich $46,2^{\circ}\left(0-60^{\circ}\right)$, wobei bei keinem Patienten ein fixierter Spitzfuß vorlag. Nach Pseudarthrosenstabilisierung konnte der durchschnittliche Bewegungsumfang im oberen Sprunggelenk von $25,3^{\circ}\left(0-60^{\circ}\right)$ auf $40,5^{\circ}\left(0-60^{\circ}\right)$ gesteigert werden.

\section{Berufliche Wiedereingliederung}

In der Pseudarthrosengruppe waren von 20 Patienten noch 16 berufstätig. Von diesen konnten 14 in ihren alten Beruf zurückkehren, 1 wurde umgeschult, 1 wurde berentet.

In der Frakturgruppe waren 19 von 24 Patienten noch berufstätig. Von diesen kehrten 14 in ihre vorher ausgeübten Berufe zurück, 2 Patienten konnten umgeschult werden, 3 wurden berentet, wobei 
Trauma Berufskrankh 2005 · 7[Suppl 1]:S58-S64

DOI 10.1007/s10039-004-0918-1

๑) Springer-Verlag 2004

\section{E. Wenzl · S. Fuchs · D. Wolter · C. Jürgens}

\section{Distale Tibiafrakturen. Erfahrungen mit einem winkelstabilen Plattensystem}

\section{Zusammenfassung}

Von September 1997-Dezember 2000 wurden bei 44 Patienten 25 Frakturen und 20 Pseudarthrosen der distalen Tibia mit einem multidirektional winkelstabilen Plattenfixateur-interne-System stabilisiert, in 92\% der Fälle minimalinvasiv in Untertunnelungstechnik. Bei 15 Frakturen wurde initial ein Fixateur externe angelegt und sekundär nach Konsolidierung der Weichteile auf das interne Implantat umgestiegen. Alle Pseudarthrosenstabilisierungen wurden mit einer autologen Spongiosaplastik kombiniert. Alle 44 Patienten konnten nach durchschnittlich 22,4 Monaten (9-48 Monaten) nachuntersucht werden. Sämtliche Frakturen und Pseudarthrosen waren durchbaut. Bei 4 Patienten traten
Plattenlagerinfekte auf, die durch frühzeitige Materialentfernung beruhigt wurden. Vollbelastung wurde bei Frakturen nach durchschnittlich 15,2 Wochen (8-26 Wochen), bei Pseudarthrosen nach 17,1 Wochen (8-52 Wochen) erreicht. Die Ergebnisse zeigen, dass sich das multidirektional winkelstabile Fixateur-interne-System in Verbindung mit minimalinvasiver Technik und dem vorgestellten Konzept für die Behandlung von Frakturen und Pseudarthrosen der distalen Tibia sehr bewährt hat.

\section{Schlüsselwörter}

Winkelstabiles Plattensystem · Fixateur interne Distale Tibia Pseudarthrosen

\section{Fractures and pseudarthroses of the distal tibia. Experience with a fixed-angle internal fixator system}

\begin{abstract}
From September 1997 to December 2000, 25 fractures and 20 psaeudarthroses in 44 patients were treated by fixed-angle plating; in $92 \%$ of these cases a minimally invasive tunneling technique was used. Fifteen fractures had initially been stabilized by means of an external fixator, the internal fixator being implanted as a second step once consolidation of the soft tissue had been achieved. Revision of a pseudarthrosis was always combined with autologous bone grafting. Follow-up examinations were possible in all 44 patients after an average of 22 (range 9-48) months, and all fractures and pseudarthroses were found to have healed. No secondary loss of reduction or implant
\end{abstract}

failure was seen. In 4 patients deep infection at the plate occurred, but was successfully treated by early implant removal. Full weight-bearing was possible after a mean of 15.2 (range 8-26) weeks for the fracture patients and of 17.1 (mean; range 8-52) weeks for the pseudarthrosis patients. These results show that the multidirectional fixed-angle internal plate fixator implanted by a minimally invasive technique has proved very reliable in the treatment of fractures and pseudarthroses of the distal tibia.

\section{Keywords}

Fixed-angle plates · Pseudarthrosis . Nonunion $\cdot$ Internal fixator $\cdot$ Distal tibia
2 ein schweres Polytrauma erlitten hatten und 1 älter als 60 Jahre war.

\section{Komplikationen}

An postoperativen Komplikationen traten 1 tiefe Beinvenenthrombose nach Polytrauma, 1 Achsfehler (Außenrotationsfehler von $15^{\circ}$ ), 3 Plattenlagerinfekte und 1 Osteitis auf.

In keinem Fall kam es zu einem sekundären Korrekturverlust oder zu einem $\mathrm{Ma}$ terialversagen.

In der Frakturgruppe fanden sich 2 Plattenlagerinfekte. In beiden Fällen handelte es sich um offene Frakturen, die zunächst im Fixateur externe stabilisiert worden waren. Bei 1 Patienten kam es erst nach 26 Wochen zu einer blanden Fistel ohne Keimnachweis bei bereits verheilter Fraktur. Die Situation konnte durch eine vorzeitige Materialentfernung beruhigt werden. Bei $1 \mathrm{~Pa}$ tienten wurde dem sehr dünnen Weichteilmantel im distalen Tibiabereich in Kombination mit einer peripheren AVK Stadium IIa nach Fontaine nicht Rechnung getragen. Hier kam es zu einer Wunddehiszenz mit frei liegender Platte, sodass der TiFix nach 10 Wochen bei fortschreitender Konsolidierung entfernt werden musste. Die Fraktur heilte im Unterschenkelgehapparat ohne Infekt verzögert aus.

Bei den Pseudarthrosen fanden sich ebenfalls 2 Plattenlagerinfekte. In beiden Fällen war zum Zeitpunkt des Auftretens der Fisteln bereits eine knöcherne Durchbauung vorhanden, zum Zeitpunkt der TiFix-Implantation hatte zudem ein positiver Keimbefund, also eine Infektpseudarthrose, vorgelegen. Im ersten Fall konnte der Infekt durch Materialentfernung nach 7 Monaten beruhigt werden. Im 2. Fall musste nach initialer Infektberuhigung durch Materialentfernung 1 Jahr später bei erneuter Fistelung ein Sequester entfernt werden, sodass hier von einer Osteitis auszugehen ist.

\section{Fallbeispiele}

Anhand von 2 klinischen Fällen wird das Prinzip der TiFix-Implantation in minimalinvasiver Technik bei Frakturen und Pseudarthrosen der Tibia dargestellt. 


\section{Fall 1}

Ein 28-jährigen Maurer zog sich bei einem Gerüststurz eine zweitgradig offene distale Unterschenkelfraktur Typ C3 mit Gelenkbeteiligung zu. Nach initialer Stabilisierung im Fixateur externe fand sich nach 7 Monaten eine instabile Varuspseudarthrose (• Abb. 2a).

Bei $20^{\circ}$ Varusfehlstellung wurde nach Entfernung der Schraube im Pilon eine Stabilisierung mit dem TiFix in minimalinvasiver Technik in Verbindung mit einer autologen Spongiosaplastik durchgeführt (• Abb. 2b).

Es konnte eine vollständige Fehlstellungskorrektur erreicht werden.

Die Pseudarthrose durchbaute zeitgerecht (- Abb. $2 \mathbf{c}$ ), sodass der Patient nach 12 Wochen wieder voll belasten konnte. Nach 6 Monaten kehrte er in seinen Beruf als Dachdecker zurück.

\section{Fall 2}

Ein 40-jähriger Chemiearbeiter zog sich bei einem schweren Distorsionstrauma eine langstreckige Torsionsfraktur des Unterschenkels mit Beteiligung des Pilon tibiale (Typ C1 nach AO) zu. Die Weichteilsituation erlaubte die sofortige interne Stabilisierung am Unfalltag. Deshalb wurde nach Verplattung der Fibula mit Wiederherstellung der Länge zunächst das Pilon tibiale perkutan verschraubt und anschließend die Tibia in minimalinvasiver Technik mit einem 180 mm langen TiFix-System versorgt. Am postoperativen Röntgenbild erkennt man gut, dass der Plattenfixateur interne dem Knochen nicht anliegen muss (• Abb. 3a).

Die klinischen Bilder am 14. postoperativen Tag zeigten völlig reizlose Weichteile ohne wesentliche Schwellung nach minimalinvasiver Osteosynthese (• Abb. 3b).

Die große Überbrückungsstrecke war nach 4 Monaten knöchern überbaut. Der Patient hatte nach 12 Wochen mit der Vollbelastung begonnen.

Das Material ist inzwischen entfernt. Der Patient ist bei freier Beweglichkeit im OSG in seinen alten Beruf zurückgekehrt.

\section{Diskussion}

Für die Behandlung von Tibiafrakturen wird in den meisten Studien der Marknagel favorisiert $[1,3,4,10,13,15,19,20,22]$. Die Marknagelindikation wurde immer weiter ausgedehnt, bis hin in das proxima- le [18] und distale Fünftel [19], wobei von einigen Autoren die Verwendung von Pollerschrauben angegeben wurde, um postoperative Korrekturverluste zu vermeiden $[12,18]$, die aus der trompetenförmigen Aufweitung des Markraums im proximalen und distalen Metaphysenbereich resultieren.

Richter et al. [19] gaben bei 50 Patienten mit distalen Frakturen, die mit unaufgebohrten Marknägeln versorgt wurden, 2 sekundäre Repositionsverluste, 1 Pseudarthrose und 1 Perforation des Marknagels durch das Pilon tibiale in das Sprunggelenk an. Müller et al. [15] berichteten nach ungebohrter Marknagelosteosynthese bei 69 offenen Frakturen, die ausschließlich im proximalen und distalen Drittel lokalisiert waren, über 6 relevante Fehlstellungen. Aber auch nach konventioneller Plattenosteosynthese von proximalen und distalen Frakturen sind sekundäre Fehlstellungen beschrieben $[2,17]$.

Im untersuchten Patientenkollektiv kam es bei der Stabilisierung von distalen Tibiafrakturen und -pseusarthrosen mit dem TiFix-System zu keinen sekundären Korrekturverlusten. Bei 40\% offenen Frakturen und $25 \%$ mehrfach und polytraumatisierten Patienten handelte es sich hier um ein negativ selektioniertes Patientengut. Auch die Dauer der 20 Pseudarthrosen mit 13,2 Monaten (6-66 Monaten) und die Anzahl der Voroperationen von durchschnittlich 3,4 (1-10) zeigen, dass es sich überwiegend um Problemfälle gehandelt hat. Alle 44 nachuntersuchten Patienten erreichten einen knöchernen Durchbau, wobei nur bei 1 einzigen Patienten mit einer Infektpseudarthrose ein 2. Eingriff (nochmalige Spongiosaplastik) nötig war.

Müller et al. [15] gaben bei der ungebohrten Marknagelung von 69 offenen Frakturen eine Pseudarthroserate von $12 \%$ an, wobei nahezu ausschließlich das distale Drittel betroffen war. Die Häufung der Pseudarthrosen im distalen Drittel begründeten Menck et al. [14] damit, dass die distale Tibia nur von einem periostalen $\mathrm{Ge}$ fäß ernährt wird, wohingegen die übrigen Tibiasegmente über mehrere nutritive $\mathrm{Ge}$ fäße verfügen.

Bei dem Versuch, 32 Pseudarthrosen nach gebohrter Marknagelung durch Nagelwechsel zu sanieren, hatten Court-Brown
Tabelle 2

Versorgungsstrategien bei distalen Tibiafrakturen

\begin{tabular}{ll}
\hline Anzahl Frakturen & 25 \\
\hline Primärimplantation & 10 \\
\hline $\begin{array}{l}\text { Zeit Unfall-Operation [Tage] } \\
\text { - Durchschnitt } \\
\text { - Spannweite }\end{array}$ & 4,5 \\
\hline Primär Fixateur externe & $0-15$ \\
\hline $\begin{array}{l}\text { Liegedauer Fixateur [Tage] } \\
\text { - Durchschnitt } \\
\text {-Spannweite }\end{array}$ & 33 \\
\hline $\begin{array}{l}\text { Fixateurfreies Intervall } \\
\text { Dauer Intervall [Tage] } \\
\text { - Durchschnitt } \\
\text {-Spannweite }\end{array}$ & $3-86$ \\
\hline $\begin{array}{l}\text { Minimalinvasive } \\
\text { Operationstechnik }\end{array}$ & 19,3 \\
\hline Autologe Spongiosaplastik & $9-31$ \\
\hline Septopalketten & 25 \\
\hline Positiver Keimnachweis & 16 \\
\hline
\end{tabular}

Tabelle 3

Patientendaten bei distalen Tibiapseudarthrosen (bei Vorimplantaten Mehrfachnennungen möglich)

\begin{tabular}{ll} 
Zeitraum der Versorgung & $\begin{array}{l}12 / 97-12 / 00 \\
\text { Patientenzahl }\end{array}$ \\
& $\begin{array}{l}20(69, \\
\left.140^{7}\right)\end{array}$ \\
\hline $\begin{array}{l}\text { Alter [Jahre] } \\
\text { - Durchschnitt } \\
\text { - Spannweite }\end{array}$ & 38,6 \\
\hline $\begin{array}{l}\text { Dauer der Pseudarthrose } \\
\text { [Monate] }\end{array}$ & $21-55$ \\
\hline $\begin{array}{l}\text { - Durchschnitt } \\
\text { - Spannweite }\end{array}$ & 13,2 \\
\hline
\end{tabular}

\begin{tabular}{lc}
$\begin{array}{l}\text { Anzahl der Voroperationen } \\
\text { - Durchschnitt }\end{array}$ & \multicolumn{2}{l}{3,4} \\
- Spannweite & $1-10$ \\
\hline $\begin{array}{l}\text { Vorimplantate } \\
\text { - Marknagel }\end{array}$ & 4 \\
- Fixateur externe & 21 \\
- Platte & 5 \\
- Sonstige & 1 \\
\hline $\begin{array}{l}\text { Primärer Gefäß-Nerven- } \\
\text { Schaden }\end{array}$ & 4 \\
\hline $\begin{array}{l}\text { Fraktur primär offen } \\
\text { Unfallart } \\
\text { - Monotrauma } \\
\text { - Mehrfachverletzung } \\
\text { - Polytrauma }\end{array}$ & 15 \\
\hline
\end{tabular}




\section{Neue Osteosynthesetechniken}
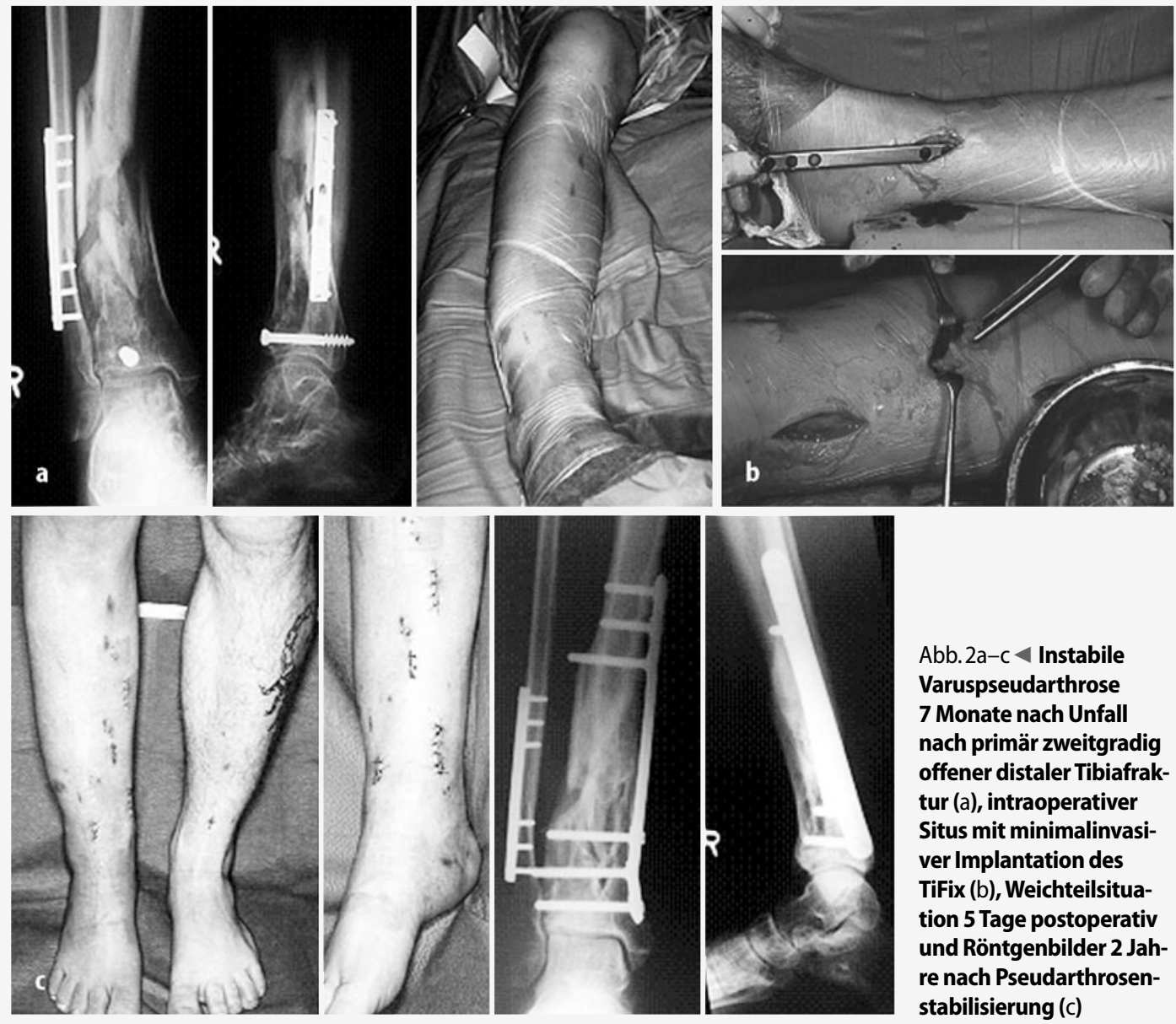

Abb. 2a-c $<$ Instabile

Varuspseudarthrose

7 Monate nach Unfall

nach primär zweitgradig

offener distaler Tibiafrak-

tur (a), intraoperativer

Situs mit minimalinvasi-

ver Implantation des

TiFix (b), Weichteilsitua-

tion 5 Tage postoperativ

und Röntgenbilder 2 Jah-

re nach Pseudarthrosen-

stabilisierung (c)
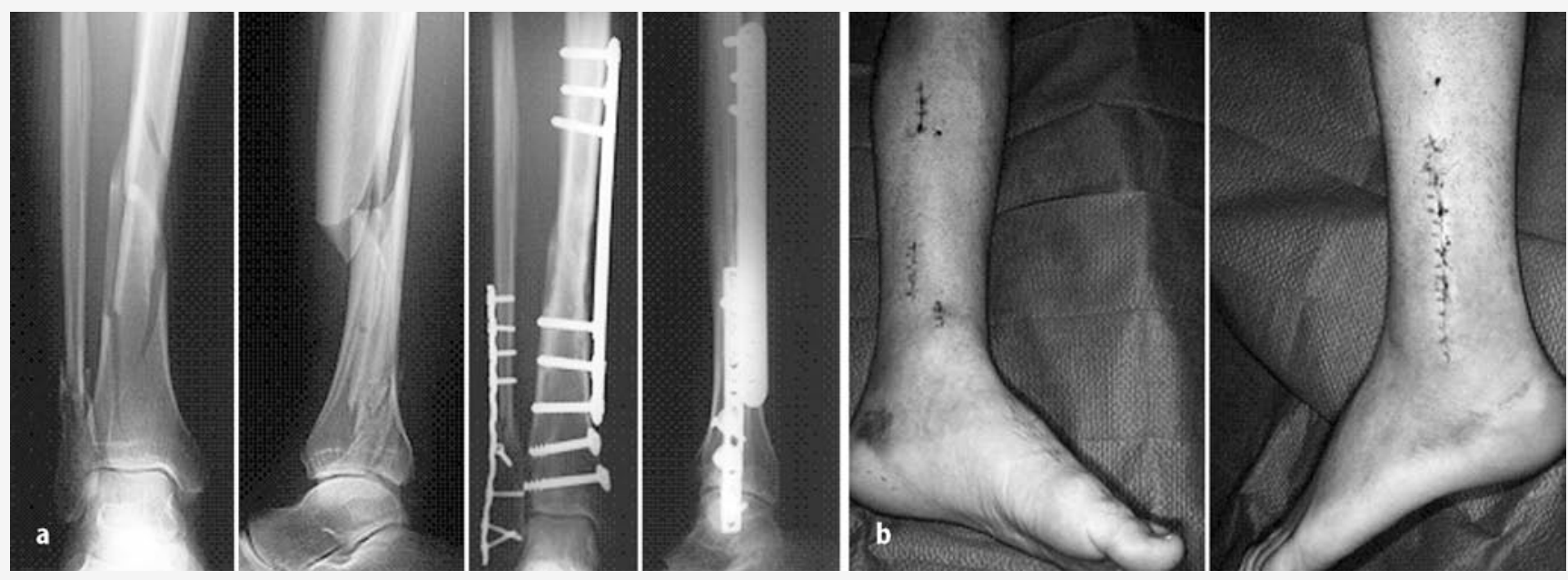

Abb. 3a, b $\Delta$ Langstreckige geschlossene distale Tibiaspiralfraktur mit Gelenkbeteiligung vor und nach Versorgung mit TiFix und Fibulaplatte(a), klinisches Bild 14 Tage nach Operation vor Fadenzug (b)

et al. [4] in 4 Fällen (12\%) keinen Erfolg. Dagegen erzielten Megas et al. [13] mit der aufgebohrten Marknagelung aseptischer Tibiapseudarthrosen eine 100\%ige Heilungsrate innerhalb von 6 Monaten, wobei nur in 3 Fällen autologe Spongiosa angelagert wurde. Sie gaben eine Vorbehandlung im Fixateur externe bei 36 von 50 Patienten mit Pseudarthrosen an (72\%). Auch im untersuchten Kollektiv war der Fixateur externe mit $60 \%$ die häufigste primäre Stabilisierungsform in der Pseudarthrosengrup- pe. Helfet et al. [9] sahen dagegen eine vorausgegangene Fixateur-externe-Behandlung, Fehlstellungen oder proximale und distale Pseudarthrosen als Kontraindikation zur Pseudarthrosenstabilisierung mit einem Marknagel an. Sie berichteten über 
33 Patienten mit fehlgestellten Pseudarthrosen, die sie mittels Plattenosteosynthese therapiert hatten. Sie gaben eine indirekte Reposition über intraoperativ angelegte externe Fixateure an, um eine möglichst schonende Applikation der Platte zu gewährleisten. Auf diese Weise konnten nach durchschnittlich 4 Monaten (2-18 Monaten) alle 33 Pseudarthrosen zur Ausheilung gebracht werden, wobei in 29 von 33 Fällen $(87,9 \%)$ eine autologe Spongiosaplastik durchgeführt wurde. Dies bestätigt das Konzept der grundsätzlichen Spongiosatransplantation bei der Stabilisierung von Pseudarthrosen im untersuchten Patientenkollektiv.

Bezüglich des Zeitpunkts der erreichten Vollbelastung kann sich die winkelstabile Plattenfixateur-interne-Osteosynthese durchaus mit den Ergebnissen nach Marknagelung vergleichen. In der Problemzone der distalen Frakturen wurde im untersuchten Patientenkollektiv die Vollbelastung nach durchschnittlich 15,2 Wochen (7-26 Wochen) erreicht. In der Studie von Richter et al. [19] war nach ungebohrter Marknagelung eine Vollbelastung nach 15,6 Wochen (6-66 Wochen) möglich. Auch für die Pseudarthrosentherapie sind die Zeiträume von 13-17 Wochen genau im Rahmen dessen, was nach Marknagelung erreicht wird [4, 9, 13].

Insgesamt entstand der Eindruck, dass eine raschere Vollbelastung bei dem sehr stabilen Implantat möglich ist, wie einige Fälle von Patienten zeigten, die „zu früh“ belastet haben, ohne dass es zu einem Materialbruch gekommen wäre.

Alle Patienten der Frakturgruppe erreichten wieder ein freies Bewegungsausmaß im Kniegelenk ohne Knieschmerzen. Im Gegensatz hierzu beschrieben Richter et al. [19] nach ungebohrter Unterschenkelmarknagelung distaler Frakturen bei 12 von 47 Patienten ein Streckdefizit bis $20^{\circ}$ im Kniegelenk.

Die nach Marknagelung beschriebenen vorderen Knieschmerzen, die in einer Studie von Toivanen et al. [24] selbst nach Materialentfernung noch bei fast 70\% der Patienten aufgetreten waren, konnten naturgemäß bei keinem Patienten beobachtet werden.

Die Infektionsrate liegt mit 2 bei 25 Frakturen $(8 \%)$ und 2 bei 20 Pseudarthrosen (10\%) im Bereich der in der Literatur angegebenen Zahlen (Court-Brown et al. [4] 12,1\% nach gebohrter Marknagelung von
Pseudarthrosen; Megas et al. [13] 2\% nach gebohrter Marknagelung von Pseudarthrosen; Helfet et al. [9] 15\% nach konventioneller Plattenosteosynthese von Pseudarthrosen; Pugh et al. [17] 12,5\% nach ORIF von Pilon-tibiale-Frakturen; in der Metaanalyse von Bhandari et al. [1] 10,2\% tiefe und $6,0 \%$ oberflächliche Infektionen nach ungebohrter Markangelung offener Tibiaschaftfrakturen; Siebert et al. [23] 2,5\% nach primärer Fixateur-externe-Anlage und sekundärem Umstieg auf konventionelle Plattenosteosynthese). Unter diesen Zahlen sind alle Infektionen subsumiert, also auch solche, die zur Amputation aufgrund des Infekts geführt haben.

Bei Analyse der 4 Infektionen zeigte sich, dass 3 Plattenlagerinfekte im Sinn von Fistelungen erst zu einem späten Zeitpunkt aufgetreten waren - als die Fraktur bzw. die 2 Pseudarthrosen schon knöchern durchbaut waren. In allen 3 Fällen (1 offene Fraktur mit sekundärem Umstieg nach Fixateur-externe-Anlage und 2 Pseudarthrosen) lag ein positiver Keimnachweis bei TiFix-Implantation vor.

Bei einem Patienten mit pAVK und sehr dünnem Weichteilmantel trat nach sekundärem Umstieg vom Fixateur externe auf das TiFix-System bereits 1 Woche postoperativ eine Wunddehiszenz auf. Die Indikation für dieses Vorgehen ist kritikwürdig und hier sind sicher die Grenzen des Verfahrens durch das Weichteil aufgezeigt worden.

\section{Fazit für die Praxis}

Bei der Behandlung von Frakturen und Pseudarthrosen der Tibia mit dem multidirektional winkelstabilen Fixateur-interneSystem haben sich folgende Aspekte als wichtig erwiesen:

\section{- Das Konzept der initialen Fixateur-ex- terne-Stabilisierung mit sekundärem Umstieg auf den winkelstabilen Patten- fixateur hat sich bewährt. Durch die mi- nimalinvasive Operationstechnik ist die Weichteilschädigung eher geringer als beim Marknagel, da die Kniegelen- kregion nicht mitbetroffen ist. \\ - Mögliche Komplikationen der Markna- gelung wie Schaftsprengungen, Hit- zenekrosen beim Aufbohren [16] und Fett- oder Luftembolien [23] können so vermieden werden.}

- Trotz einer Rate von $\mathbf{5 0 \%}$ offenen Frakturen kam es zu keiner einzigen Osteitis, sondern nur zu 2 blanden Plattenlagerinfekten. Diese sind in der Regel unproblematischer zu therapieren als eine Markraumphlegmone, wie sie nach Marknagelung auftreten kann.

- Durch die hohe Primärstabilität des winkelstabilen Plattenfixateur interne für Biege- und Torsionsbelastungen, die über einen langen Zeitraum anhält, können auch infizierte Pseudarthrosen und Defektfrakturen ohne sekundäre Korrekturverluste zur knöchernen Ausheilung gebracht werden.

- Für die Behandlung von Pseudarthrosen jeder Lokalisation am Tibiaschaft ist die hohe Stabilität des Plattenfixateur interne in Verbindung mit einer minimalinvasiven Operationstechnik und der autologen Spongiosaplastik ein Garant für eine sehr hohe knöcherne Konsolidierungsrate nach dem Ersteingriff.

\section{Korrespondierender Autor}

\section{Dr. M. E. Wenzl}

Klinik für Unfallchirurgie,

Universitätsklinikum Schleswig-Holstein, Campus Lübeck,

Ratzeburger Allee 160, 23538 Lübeck

E-Mail: michael.wenzl@chirurgie.uni-luebeck.de

Interessenkonflikt: Keine Angaben

\section{Literatur}

1. Bhandari M, Guyatt GH, Swiontkowski MF et al. (2001) Treatment of open fractures of the shaft of the tibia. J Bone Joint Surg Br 83: 62-68

2. Bono CM, Levine RG, Rao JP et al. (2001) Nonarticular proximal tibial fractures: treatment options and decision making. J Am Acad Orthop Surg 9: 176186

3. Court-Brown CM, Christie J, McQueen MM (1990) Closed intramedullary tibial nailing: its use in closed and type I open fractures. J Bone Joint Surg Br 72: 605-611

4. Court-Brown CM, Keating JF, Christie J et al. (1994) Exchange intramedullary nailing. J Bone Joint Surg Br 77: 407-411

5. Fuchs S, Wolter D, Kranz HW et al. (2001) Titan-Fixateur-interne-Systeme mit multidirektionaler Winkelstabilität im Unterschenkel- und Fußbereich. Trauma Berufskrankh [Suppl 4] 3: S425-428

6. Gerber A, Ganz R (1998) Combined internal and external osteosynthesis. A biological approach to the treatment of complex fractures of the proximal tibia. Injury 29: S-C22-S-C28

7. Goldhahn S, Moser R, Bigler R et al. (2000) Behandlungsmethoden und -ergebnisse von Tibiaschaftfrakturen in der Schweiz. Swiss Surg 6: 315-322 
8. Gruner A, Hockertz T, Reilmann H (2000) Die proximale Tibiafraktur. Unfallchirug 103: 668-684

9. Helfet DL, Jupiter JB, Gasser S (1992) Indirect reduction and tension-band plating of tibial non-union with deformity. J Bone Joint Surg Am 74: 12861297

10. Keating JF, Blachut PA, O'Brien PJ et al. (2000) Reamed nailing of Gustilo grade-IIIB tibial fractures. J Bone Joint Surg Br 82: 1113-1116

11. Kranz H-W, Wolter D, Queitsch C et al. (1999) Therapie von Pseudarthrosen, Fehlstellungen und Frakturen im Unterschenkelbereich mit einem Titanfixateur interne. Trauma Berufskrankh 1:356-360

12. Krettek C, Miclau T, Schandelmaier P et al. (1999) The mechanical effect of blocking screws („Poller screws") in stabilizing tibia fractures with short proximal or distal fragments after insertion of small diameter intramedullary nails. J Orthop Trauma 13: 550-553

13. Megas $P$, Panagiatopoulos $E$, Skriviliotakis $S$ et al. (2001) Intramedullary nailing in the treatment of aseptic tibial non-union. Injury 32: 233-239

14. Menck J, Bertram C, Lierse W (1992) Sectorial angioarchitecture of the human tibia. Acta Anat 143: 67-73

15. Müller CA, Dietrich M, Morakis M et al. (1998) Klinische Ergebnisse der primären Marknagelosteosynthese mit dem unaufgebohrten AO/ASIF Tibiamarknagel von offenen Tibiaschaftfrakturen. Unfallchirurg 101: 830-837

16. Ochsner PE, Baumgart F, Kohler $G$ (1998) Heat-induced segmental necrosis after reaming of one humeral and two tibial fractures with a narrow medullary canal. Injury 29: B1-10

17. Pugh KJ, Wolinsky PR, McAndrew MP et al. (1999) Tibial pilon fractures: a comparison of treatment methods. J Trauma 47: 937-941

18. Ricci WM, O'Boyle M, Borelli J et al. (2001) Fractures of the proximal third of the tibial shaft treated with intramedullary nails and blocking screws. J Orthop Trauma 15: 264-270

19. Richter D, Hahn MP, Laun RA et al. (1998) Der sprungggelenksnahe Unterschenkelbruch. Chirurg 69: $563-570$

20. Schandelmaier P, Kretteck C, Rudolf J et al. (1997) Vorteile des unaufgebohrten Tibianagels im Vergleich mit Fixateur externe bei der Behandlung von Grad 3 B offenen Tibiaschaftfrakturen. Unfallchirurg 100: 286-293

21. Seide K, Wolter D, Kortmann HR (1999) Fracture reduction and deformity correction with the hexapod Ilizarow fixator. Clin Orthop 363: 186-195

22. Shannon FJ, Mullet H, O'Rourke K (2002) Unreamed intramedullary nailing versus external fixation in grade III open tibial fractures. J Trauma 52: 650-654

23. Siebert $\mathrm{CH}$, Lehrbass-Sokeland KP, Rinke $\mathrm{F}$ et al. (1997) Compression plating of tibial fractures following primary external fixation. Arch Orthop Trauma Surg 116: 390-395

24. Toivanen JAK, Väistö O, Kannus P et al. (2002) Anterior knee pain after intramedullary nailing of fractures of the tibial shaft. J Bone Joint Surg Am 84: 580-585

25. Wolter D, Schümann U, Seide K (1999) Universeller Titanfixateur interne. Entwicklungsgeschichte, Prinzip, Mechanik, Implantatgestaltung und operativer Einsatz. Trauma Berufskrankh 1:307-319 